\title{
A25 ANTI-ATP SYNTHASE AUTOANTIBODIES ALTER ENDOTHELIAL CELL (EC) SURVIVAL BY DISRUPTING PH REGULATION IN VASCULIT
}

C Jamin, J-E Alard, J-0 Pers, P Youinou EA 2216 'Immunologie et Pathologie' and IFR 148 ScInBioS, Université de Brest, and Université Européenne de Bretagne, Brest, France

\subsection{6/ard.2010.129577y}

Background B-cell clusters represent genuine germinal centres (GCs) in the SGs of SS patients, but their majority display transitional and marginal zone (MZ)-like B cell characteristics. We thus asked the question as to whether TLR9 stimulation that occurs during inflammatory reaction could influence such an aberrant B-cell differentiation.

Methods Following laser microdissection, TLR expression was measured in B-cell aggregates using quantitative RT-PCR. As controls, transitional and mature B cells from cord blood and tonsils were FACS sorted and stimulated with CpG-ODN. CFSE enabled to evaluate their proliferation, they were also analysed by flow cytometry to determine their activation status. Differentiation into Ig-secreting cells was demonstrated by ELISA.

Results Transitional B-cell aggregates of SGs expressed high level of TLRs whereas real GC lacked TLR expression. In vitro, TLR9 stimulation induced proliferation of transitional $B$ cells, activation and matu ration as MZ B cells (Notch2high, CD21high, IgMhigh, IgDlow, CD23low, CD27-). Mature B cells proliferated also but differentiated toward a follicular pathway (Notch2low, IgMlow, IgDlow, CD23low, CD27+). Production of Ig was observed in both B-cell supernatants.

Conclusion High TLR level in transitional SG B-cell aggregates indicates how highly sensitive to inflammatory stimuli they are. Immature B cells can differentiate into Ig-secreting cells following TLR9 stimulation through a MZ-like maturation pathway without entering the GCs. In contrast, mature $B$ cells differentiate towards a classical follicular pathway. Whether one or both differentiation pathway generate autoreactive B cells in SGs remains to be established in SS patients 